\title{
openheart The 7-year teesside experience of primary prevention ICD indications following primary PCI (PPCI) and the potential impact of a change in NICE guidance
}

Thanh Trung Phan, ${ }^{1}$ Saima Khan, ${ }^{1}$ Muhammad Muzaffar Mahmood, ${ }^{1}$ Sudha Mani, ${ }^{2}$ Vineet Wadehra, ${ }^{3}$ Mark de Belder, ${ }^{1}$ Andrew Thornley, ${ }^{1}$ Simon James, ${ }^{1}$ Nicholas J Linker, ${ }^{1}$ Andrew J Turley ${ }^{1}$

To cite: Phan TT, Khan S, Mahmood MM, et al. The 7-year teesside experience of primary prevention ICD indications following primary $\mathrm{PCl}(\mathrm{PPCl})$ and the potential impact of a change in NICE guidance. Open Heart 2015;2: 000153. doi:10.1136/openhrt-2014000153

Received 6 June 2014 Revised 18 November 2014 Accepted 26 November 2014

\section{(a) CrossMark}

\begin{abstract}
${ }^{1}$ James Cook University Hospital, Middlesbrough, UK ${ }^{2}$ Darlington Memorial Hospital, County Durham, UK ${ }^{3}$ University Hospital of North Tees, Stockton-on-Tees, UK
\end{abstract}

Correspondence to Dr Thanh Phan, The James Cook University Hospital, Marton Road, Middlesbrough.

TS4 3BW, UK;

ttpquang@hotmail.com

\section{ABSTRACT}

Introduction The recovery of LV function in patients with severe LV impairment in the acute phase following primary percutaneous coronary intervention (PPCI) is not well established. The indication for a primary prevention ICD post-STEMI is dependent on which screening guidance, NICE or ESC, is followed. The potential impact of the new NICE guidance is estimated.

Methods We performed a retrospective analysis of all patients presenting with a STEMI over a 7-year period (2005-2012) treated with PPCI to determine inhospital mortality, LV function at index presentation, at 3 months and the predicted primary prevention ICD implantation rate using NICE (TA095) and ESC 2006 guidelines. Predicted implant rates using the new NICE guidance (TA314) and actual implantation rates were also assessed.

Results 3902 patients with a mean age of $65 \pm 13$ years underwent $\mathrm{PPCl}$. Of those patients surviving until discharge, $332(10 \%)$ had LVEF $\leq 35 \%$. 254 of 332 patients $(76 \%)$ with a severely impaired ventricle were followed up at participating centres. 210 of 254 (83\%) patients had a repeat echocardiogram within 3 months post-Ml; among these patients, $89(42 \%)$ remained to have LVEF $\leq 35 \%$. The number of patients fulfilling NICE and ESC criteria for primary prevention ICD implantation was $14(16 \%)$ and $84(94 \%)$, respectively. The actual number of patients receiving an ICD was 17 $(19 \%)$. The number of patients fulfilling the new NICE (TA314) guidance was 84 (94\%).

Conclusions A small proportion of patients with STEMIs undergoing PPCI have a severely impaired LV systolic function. A large proportion of these patients will have improved LV systolic function at 3 months. There is a five-fold difference in the predicted ICD implantation rates depending on which guidance is followed-NICE versus ESC. The potential impact of the new NICE (TA314) guidance on ICD implantation will be a significant increase in ICD implantation rates.

\section{KEY MESSAGES}

What is already known about this subject?

- Despite the widespread introduction of primary percutaneous coronary intervention (PPCl), the recovery of left ventricular (LV) function in those with severe LV impairment in the acute phase is not well established. There are limited data on the recovery of LV function following PPCI in the modern era with modern medical therapy. As far as we are aware, there are no estimates on primary ICD implantation rates following PPCI depending on whether National Institute of Health and Care Excellence (NICE) or European society of cardiology (ESC) guidance is used. There are no data on the impact of the new NICE (TA314) guidance on the primary ICD implantation rates in the post-PPCl population.

What does this study add?

- This study adds information on the impact of the new NICE (TA314) guidance on the predicted ICD implantation rates. We also provide further information on the evolution of the LV ejection fraction following $\mathrm{PPCl}$ and therefore the potential need for ICD implantation.

How might this impact on clinical practice?

- The new NICE guidance is likely to increase the ICD implantation rates, particularly in the post-PPCI population.

- We also provide further information on the evolution of the LV ejection fraction following PPCI which is important when assessing needs for ICD therapy.

\section{INTRODUCTION}

ST-elevation myocardial infarction (STEMI) can cause significant deleterious effects to left ventricular (LV) systolic function. The degree of LV systolic dysfunction (LVSD) has 
important implications for prognosis of patients. LVSD, especially if severe, increases the risk of sudden cardiac death (SCD) following MI. ${ }^{1}$ Several randomised controlled trials have shown a beneficial prognostic effect of implantable cardioverter defibrillators (ICD) in prevention of SCD following MI. ${ }^{2-4} \mathrm{~A}$ number of current society guidelines (European society of cardiology (ESC) and Heart rhythm society (HRS)) include severe impairment of LV systolic function (ejection fraction (EF) $35 \%$ or less) as an essential criterion for identifying patients in whom ICD for the primary prevention of SCD should be considered. ${ }^{5}$ The ESC guidance state that patients should be on optimal medical therapy (OMT) for 3 months and those patients should be re assessed by 3 months postrevascularisation. ${ }^{5}$

Significant advances have been made in recent years in the management of MI including the routine use of primary PCI for patients with STEMI. Primary PCI, now considered a standard of care for all patients with STEMI, reduces mortality and morbidity when compared to thrombolysis. ${ }^{6}$ In an earlier meta-analysis of 23 trials including 7739 thrombolytic-eligible patients with ST-segment elevation, acute myocardial infarction (AMI) randomly assigned to primary percutaneous transluminal coronary angioplasty (12 trials used stents) or thrombolytic therapy noted a reduction in death, nonfatal reinfarction, stroke and the combined end point of death, non-fatal reinfarction and stroke. ${ }^{6}$ It is also recognised that LV systolic function following MI is not a static phenomenon with evidence of a variable degree of improvement in LV systolic function in the weeks following the acute MI. ${ }^{7}$ This improvement in LV systolic function after the MI may provide an explanation for the lack of a clear benefit with routine early (6-40 days) implantation of ICD in the DINAMIT trial. Importantly, LV function was reassessed in only $47 \%$ of patients in the DINAMIT trial. With the widespread introduction of PPCI, data on evolution of LV function in the first 3 months after the acute MI, treated with primary angioplasty, are limited.

There is a marked geographical variation in the rates of implantation of cardioverter defibrillators (ICDs), both within different regions of the UK and between different European countries. ${ }^{8} 9$ Furthermore, significant differences exist between the current National Institute of Health and Care Excellence (NICE) ${ }^{10}$ and European society of cardiology (ESC) $)^{5}$ guidance for implantation of ICD for primary prevention of SCD in patients with previous MI. The new proposed changes in NICE guidance $^{11}$ on ICD has a restriction based on QRS duration difference to ESC guidance (table 1).

We sought to investigate the prevalence of severely impaired LV systolic function $(\mathrm{EF} \leq 35 \%)$ in patients who underwent PPCI for STEMI at our regional centre at index presentation and at 3 months. We also determined our ICD implantation rate and the predicted primary prevention ICD implantation rates using existing NICE and ESC guidance. We compared the predicted implant rates (using NICE (2006) guidance, ESC guidance) versus actual implant rates and then assessed the potential impact of using the new NICE (TA314) guidance.

Table 1 An outline of NICE and ESC guidance on the use of ICD for primary prevention

\begin{tabular}{lll}
\hline NICE guidance (2006) & ESC guidance (2006) $^{\mathbf{5}}$ & NICE guidance (2014) $^{\mathbf{1 1}}$ \\
\hline Primary MI (more than & Primary MI (at least 40 days post-MI) AND & Patients with heart failure who have LVEF \\
4 weeks) AND; & LVEF $\leq 35 \%$ (NYHA class II or III) & $\leq 35 \%$ \\
Either & On optimal medical treatment and who have & AND; \\
LVEF $\leq 35 \%$ (no worse & reasonable expectation of survival with a good & NYHA class I-III symptoms, and a QRS \\
than NYHA III) and & functional status of more than 1 year & duration of $<120$ ms \\
Non-sustained VT on & & *ICD if there is a high risk of sudden \\
holter (24 h) and & & cardiac death \\
Inducible VT (EPS) & & Or \\
OR & & NYHA class I-III symptoms and a QRS \\
LVEF $<30 \%$ and & duration of 120-149 ms without LBBB \\
QRS $>120$ ms & Or \\
& & NYHA class I symptoms \\
& and a QRS duration of 120-149 ms with & LBBB \\
& & (NYHA class II-III consider CRT-D) \\
& Or \\
& NYHA class I-III symptoms and a QRS \\
& duration $\geq 150$ ms with LBBB or no LBBB
\end{tabular}

${ }^{*} \mathrm{ICD}$ if there is a high risk of sudden cardiac death-these may include: age, sex, degree of left ventricular dysfunction, history of myocardial infarction, presence of cardiomyopathy and a range of other potential prognostic factors like B-type natriuretic peptide.

CRT-D, cardiac resynchronisation therapy with defibrillator; EPS, electrophysiology study; ICD, implantable cardioverter defibrillators; LBBB, left bundle branch block; LVEF, left ventricular ejection fraction; MI, myocardial infarction; NICE, National Institute of Health and Care Excellence; NYHA, New York Heart association; VT, ventricular tachycardia.

Bold text are key parts of the guidance. 


\section{METHODS}

\section{Patients}

The James Cook University Hospital is a tertiary cardiology referral centre serving a population of 1.6 million. A retrospective analysis of Myocardial Ischaemia National Audit Project (MINAP) data of all STEMIs presenting to the hospital over a 7-year period treated with PPCI was undertaken. Three thousand nine hundred and two patients were identified over the 7-year period from January 2005 to July 2012 inclusive.

Subjects with an EF $\leq 35 \%$ were considered for implantation of a primary prevention ICD according to either current 2006 ESC guidelines $^{5}$ or as outlined in NICE TA095. ${ }^{10}$ The outcome measures were to determine current in-hospital mortality for PPCI, LV function at index presentation and at 3 months. In addition to determining the predicted implant rates of participants identified for implantation of a primary prevention ICD in accordance with 2006 ESC guidelines ${ }^{5}$ or National Institute for Health and Care Excellence (NICE TA095), ${ }^{10}$ we compared the predicted implant rates (using current NICE guidance and ESC guidance) versus actual implant rates and then assessed the potential impact of using the new NICE guidance. When estimating ICD implantation rates, we would offer ICDs in patients with a narrow QRS.

\section{Follow-up.}

Further follow-up information regarding patients with LVEF $\leq 35 \%$ during admission $(n=332)$ was obtained from our centre $(n=147)$ and two other general district trusts $(n=107)$. In total, six hospitals contributed to the data set: The James Cook University hospital, Friarage Hospital, North Tees Hospital, Hartlepool Hospital, Darlington Hospital and Bishop Auckland Hospital. No information was available for 78 patients $(26 \%)$ because they lay outside our catchment area.

\section{Echocardiography}

All echocardiography studies were performed with a standard imaging system and software (VIVID 7, GEVingmed, Horton, Norway) by experienced a sonographers. LVEF was assessed using Simpson's biplane method using standard recommendations from the British Society of Echocardiography. ${ }^{12}$

\section{Statistics}

Data are presented as percentages for categorical variables and as means \pm SD for continuous variables. Comparisons between groups were performed using the $\chi^{2}$ test for categorical variables and the independent $\mathrm{t}$ test or Mann-Whitney $\mathrm{U}$ test for continuous variables as appropriate. A two-sided $\mathrm{p}<0.05$ was considered statistically significant. All analysis was performed using SPSS (V.17, SPSS Inc., Chicago, USA).

\section{RESULTS}

Three thousand nine hundred and two patients $(70 \%$ male) with a mean age of $65 \pm 13$ years underwent PPCI. Two hundred and sixty-four $(6.8 \%)$ patients died in hospital. Of the survivors, 3238 (83\%) had echocardiography during their index presentation with a median length of stay of $2.4 \pm 8.5$ days. In-patient echocardiography revealed normal LVEF in 1550 (48\%) patients, mild/moderately impaired in $1354(42 \%)$ patients and severely impaired in $332(10 \%)$ patients.

Patients with a severely impaired LVEF post-STEMI at the time of admission were older with a history of MI, peripheral vascular disease, cerebral vascular disease,

Table 2 Demographic variables of all post-PPCI patients with STEMI subdivided into those with LVEF $\leq 35 \%$ or LVEF $>35 \%$

\begin{tabular}{|c|c|c|c|c|}
\hline Variables & All STEMIs & In-hospital LVEF > $35 \%$ & In-hospital LVEF $\leq 35 \%$ & p Value* \\
\hline $\mathrm{N}$ & 3902 & 2904 & 332 & \\
\hline Male, n (\%) & $2725(70)$ & $2041(70)$ & $244(73)$ & 0.46 \\
\hline Age, years (range) & 65 (23-99) & $63 \pm 13$ & $65 \pm 14$ & 0.01 \\
\hline QRS duration $\geq 120 \mathrm{~ms}, \mathrm{n}(\%)$ & $98(3)$ & $67(2)$ & $31(9)$ & $<0.01$ \\
\hline Previous $\mathrm{Ml}-\mathrm{n}(\%)$ & $547(14)$ & $361(12)$ & $65(20)$ & 0.01 \\
\hline Previous angina-n (\%) & $569(15)$ & $382(13)$ & $55(17)$ & 0.36 \\
\hline Previous $\mathrm{PCl}, \mathrm{n}(\%)$ & $233(6.0)$ & $169(6)$ & $22(7)$ & 0.90 \\
\hline Previous CABG, n (\%) & $106(2.7)$ & $71(2)$ & $11(3)$ & 0.77 \\
\hline Hypertension-n (\%) & $1657(43)$ & $1210(42)$ & $139(42)$ & 0.97 \\
\hline Hypercholesterolaemia, n (\%) & $1338(34)$ & $1024(35)$ & $105(32)$ & 0.58 \\
\hline Peripheral vascular disease, $\mathrm{n}(\%)$ & $135(3.5)$ & $108(4)$ & $23(7)$ & 0.04 \\
\hline Cerebrovascular disease, $\mathrm{n}(\%)$ & $185(4.7)$ & $108(4)$ & $23(7)$ & 0.04 \\
\hline Chronic renal failure, $\mathrm{n}(\%)$ & $38(1.0)$ & $18(1)$ & $7(2)$ & 0.03 \\
\hline Heart failure, n (\%) & $30(0.8)$ & $7(0.0)$ & $12(4)$ & $<0.01$ \\
\hline Diabetes, n (\%) & $433(11)$ & $295(10)$ & 42 (13) & 0.01 \\
\hline
\end{tabular}

${ }^{*}$ Comparison of patients with LVEF $\leq 35 \%$ versus patients with LVEF $>35 \%$.

CABG, coronary artery bypass graft surgery; LVEF, left ventricular ejection fraction; MI, myocardial infarction; PCI, percutaneous coronary intervention; STEMIs, ST-elevation myocardial infarction.

Bold text are key parts of the guidance. 
Table 3 Cardiogenic shock and heart failure in patients with LVEF $\leq 35 \%$ or LVEF $>35 \%$ at presentation of their STEMI

\begin{tabular}{|c|c|c|c|}
\hline Variables & $\begin{array}{l}\text { In-hospital } \\
E F \geq 35 \%\end{array}$ & $\begin{array}{l}\text { In hospital } \\
\text { EF } \leq 35 \%\end{array}$ & p Value* \\
\hline $\mathrm{n}$ & 2904 & 332 & \\
\hline $\begin{array}{l}\text { Cardiogenic shock, } \\
\mathrm{n}(\%)\end{array}$ & $23(1)$ & $6(2)$ & 0.06 \\
\hline $\begin{array}{l}\text { Pulmonary oedema, } \\
\mathrm{n}(\%)\end{array}$ & $16(1)$ & $8(2)$ & $<0.01$ \\
\hline Cardiac arrest, n (\%) & $194(7)$ & $31(9)$ & 0.07 \\
\hline Peak troponin $\mathrm{mg} / \mathrm{dL}$ & $54 \pm 116$ & $107 \pm 173$ & $<0.01$ \\
\hline
\end{tabular}

diabetes, heart failure and chronic renal failure (table 2). These patients (LVEF $\leq 35 \%$ ) had a higher incidence of pulmonary oedema and larger troponin rise at the time of admission (table 3). Anterior infarction occurred in $55 \%$ of patients with $\mathrm{LVEF} \leq 35 \%$ during admission. At discharge, $81 \%$ and $86 \%$ of patients following STEMI were on a $\beta$-blocker or ACE inhibitors, respectively, (table 4).

Three hundred and thirty-two patients were found to have LVEF $\leq 35 \%$ during admission. Two hundred and fifty-four patients were followed up by a cardiologist at the participating centres $(76 \%)$, and therefore $24 \%$ were followed up outside our catchment area. Two hundred and ten of $254(83 \%)$ patients had an echocardiogram within 3 months post-MI; among these patients, $89(42 \%)$ remained to have LVEF $\leq 35 \%$. Of these 89 patients, $61 \%$ had anterior STEMIs. The number of patients fulfilling NICE and ESC criteria for primary prevention ICD therapy was $14(16 \%)$ and $84(94 \%)$, respectively. The actual number of patients receiving an ICD was $17(19 \%)$. The number of patients fulfilling the new NICE guidance was $84(94 \%)$ (figure 1). More patients with $\mathrm{LVEF} \leq 35 \%$ had a QRS duration $\geq 120 \mathrm{~ms}$ compared to those with LVEF $>35 \%$ (9\% vs $2 \%, \mathrm{p}<0.01$ ).

\begin{tabular}{|c|c|}
\hline Medication & All STEMIs \\
\hline Thiazide diuretic, n (\%) & $81(2)$ \\
\hline Loop diuretic, n (\%) & $903(23)$ \\
\hline Spironolactone, n (\%) & $34(1)$ \\
\hline Aldosterone antagonist & $210(5)$ \\
\hline Angiotensin inhibitor, n (\%) & $3357(86)$ \\
\hline$\beta$ blocker, $n$ (\%) & $3174(81)$ \\
\hline Statin, n (\%) & $3442(88)$ \\
\hline Aspirin, n (\%) & 3476 (89) \\
\hline Warfarin, n (\%) & $161(4)$ \\
\hline Thienopyridene, n (\%) & $3407(87)$ \\
\hline
\end{tabular}

\section{DISCUSSION}

Our study had two objectives: (1) To investigate the prevalence of severe LVSD (LVEF $<35 \%$ ) in patients with acute STEMI, treated with primary percutaneous coronary intervention (PPCI), at presentation and at 3 months after the event; and (2) to provide a prediction on the ICD implantation rates when applying the existing NICE versus ESC guidance, as well as the predicted ICD implant rates in accordance with the new NICE guidance, and to assess our actual ICD implantation rates.

The most important determinant of adverse long-term prognosis, as well as the risk of sudden arrhythmic death, in survivors of acute MI is reduced LVEF (EF $<35 \%$ ). LVSD, especially if severe, increases the risk of SCD following MI. ${ }^{1}$ Data on the prevalence of LVSD after MI are conflicting in terms of method and timing of assessment, inclusion of MI with and/or without ST segment elevation and the definition of severe LVSD. However, it has been recognised that a variable degree of improvement in LV systolic function occurs in the weeks following the acute MI in a significant number of patients. ${ }^{13}$ Data on evolution of LV function in the first 3 months after the acute MI, treated with primary angioplasty, are limited. Our study offers increased insight into the prevalence of LVSD at presentation and its subsequent evolution in patients with STEMI treated with PPCI. This is clinically important because it provides some estimation of the potential number of patients post-PPCI for STEMI who might require an ICD for primary prevention, which in turn will aid future service planning.

With respect to the evolution of LV systolic function post-MI, the Cardiac Arrhythmias and Risk Stratification after Acute Myocardial Infarction (CARISMA) showed that $23 \%$ of the total 5869 screened patients had LVEF $<40 \%$ at 3-21 days after the acute MI. Three hundred and twelve patients included in the study cohort were scheduled to undergo a repeat echocardiogram at 6 weeks to reassess LVEF. LVEF increased from the baseline value of $31 \pm 6$ to $35 \pm 10 \%$ at 6 weeks. ${ }^{7}$ In the Prevention of Sudden Cardiac Death II (PreSCD II) registry, 10612 patients were enrolled 4 weeks or later after MI in 19 cardiac rehabilitation centres in Germany. 6.9\% of all patients had an LVEF of $31-40 \%$ and $2.5 \%$ patients had an LVEF $<30 \% .{ }^{14}$ In a study of 600 patients with STEMI treated with primary angioplasty (recruited between January 1994 to January 1998), LVEF was measured at day 4 and 6 months after the MI by radionuclide ventriculography. At discharge, $36 \%$ had a low LVEF $(<40 \%)$, whereas after 6 months $27 \%$ had an $\mathrm{EF}<40 \%$. Importantly, only $44 \%$ patients were discharged on ACE-Inhibitor therapy. ${ }^{15}$

In this study, we have found that almost half of the patients who underwent PPCI for STEMI at our centre developed LV systolic dysfunction. Ten per cent of such patients had severely impaired LVSD (EF $\leq 35 \%$ ). Interestingly, LV systolic function had improved in the majority of patients at repeat echocardiogram at 3 months, with about $40 \%$ of those with severe LVSD at index presentation continuing to demonstrate severe 
Figure 1 Number of patients fulfilling each guidance for ICD therapy at 3 months post-PPCl. ICD, implantable cardioverter defibrillators; PPCl, primary percutaneous coronary intervention.

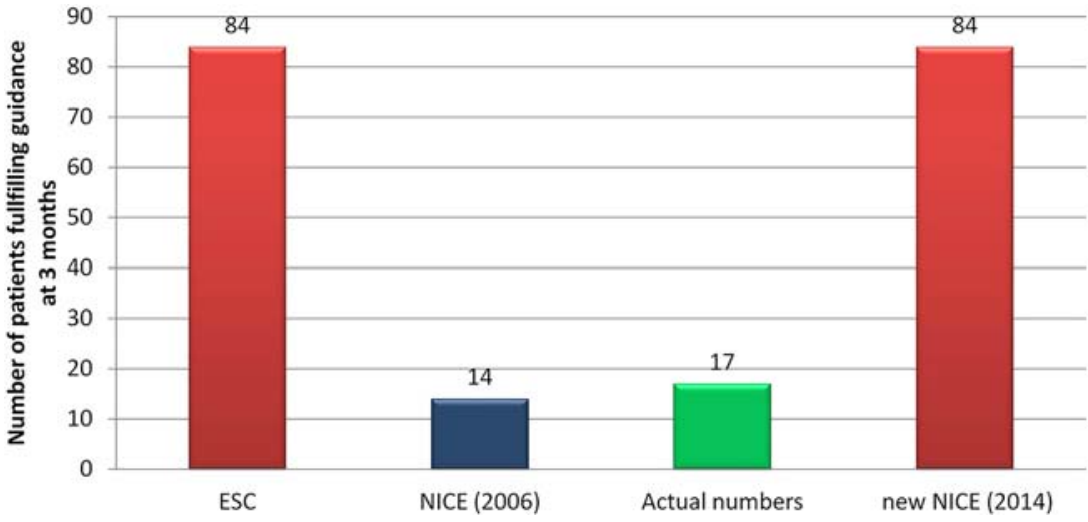

LVSD $(\mathrm{EF}<35 \%)$. Advanced age, previous MI, presence of extracardiac vascular disease and anterior location of infarct confer a higher risk of severe LVSD both immediately following the acute MI and at 3 months. This is consistent with findings noted in previous studies. ${ }^{16} 17$ Also interestingly, a significant proportion of patients $(\approx 40 \%)$ with a severely impaired LVEF at 3 months had a non-anterior infarct.

Our study presents real-life data from a single large primary PCI centre in the UK. Guidelines recommend performing an echocardiogram for reassessment of LV systolic function at least 4 weeks after the AMI; in our study, this was performed at around 3 months after the event. The rationale behind this approach was to allow time for optimisation of heart failure medications. This is consistent with the Multicenter Automatic Defibrillator Implantation Trial (MADIT) II trial which required a waiting period of at least 3 months following coronary revascularisation. ${ }^{18}$ Furthermore, we have learnt from the IRIS (Immediate Risk-Stratification Improves Survival) trial and DINAMIT (Defibrillator IN Acute Myocardial Infarction Trial) that implanting ICDs very early (within 30 or 40 days) confers no overall survival benefit following MI. ${ }^{19} 20$

The number of patients fulfilling NICE and ESC criteria for primary prevention ICD implantation was $16 \%$ and $94 \%$, respectively. The actual number of patients receiving an ICD was $19 \%$. The number of patients fulfilling the proposed NICE would have been $94 \%$. The significant differences in predicted implantation rates relate to the differences between the current $2006 \mathrm{NICE}$ and ESC guidance. ESC guidance advises an assessment of LV function at at least 40 days and that ICD implantation (with a class IA indication) should be considered in all patients with LVEF $\leq 35 \%$ who are in New York Heart association (NYHA) Functional Class II or III, are on optimal medical therapy and have a reasonable expectation of survival with a good functional status of more than 1 year. On the other hand, the 2006 NICE guidance suggests evaluation of patients with MI at least 4 weeks after the event. The NICE guidance further differentiates between EF $\leq 35 \%$ and $\mathrm{EF}<30 \%$ and suggests utilisation of ECG, Holter and electrophysiology criteria to identify high-risk patients in whom ICD implantation is recommended (table 1). It is worth mentioning here that the use of an $\mathrm{EF} \leq 35 \%$ and $\mathrm{EF}<30 \%$ to further risk stratify patients can often be difficult because the current British Society of Echocardiography (BSE) recommendation for reporting grades all LVEF $\leq 35 \%$ as severe and makes no distinction between $\mathrm{EF} \leq 35 \%$ and $\mathrm{EF}<30 \%$. $^{12}$ Nevertheless, it is fair to say that the ESC guidance for primary prevention ICD implantation is significantly more inclusive than the current $2006 \mathrm{NICE}$ guidelines. The 2012 ACCF/AHA/HRS Focused Update on ICD implantation is very similar to the ESC guidance (ie, $\mathrm{LVEF} \leq 35 \%$ due to prior $\mathrm{MI}$ in patients who are at least 40 days post-MI and are in NYHA Functional Class II or III) $i^{21}$ thus, there is a global consensus on ICD indications for primary prevention and our current NICE is out of sync with the global consensus. However, with the new NICE guidance, our practice will become more in line with the rest of the world. Interestingly, the 2013 AHA/HRS 'Appropriate Use Criteria for Implantable Cardioverter-Defibrillators and Cardiac Resynchronization Therapy' has furthermore provided some guidance on other common clinical scenarios which further broaden the scope of ICD therapy, for example, for patients with acute MI at $<40$ days, providing they also fulfil other criteria such as inducible sustained VT during EPS performed after revascularisation, within 30 days of MI. ${ }^{22}$

The new NICE guidance on ICD implantation (see table 1) differs from ESC on the requirement of a QRS duration $\geq 120 \mathrm{~ms}$, which on the surface does not appear to be significant but actually does have a significant impact on potential ICD implants. ${ }^{11}$ It important to recognise that the risk of $\mathrm{SCD}$ in those patients with QRS duration $<120 \mathrm{~ms}$ is not negligible and the mortality benefit of an ICD remains statistically significant. The all-cause mortality of patients in the MADIT II trial ICD therapy over a period of 5 years in patients with a QRS duration $>120 \mathrm{~ms}$ and those with a QRS duration $<120 \mathrm{~ms}$ was $65 \%$ and $46 \%$, respectively. ${ }^{2}$ In the The multicenter unsustained tachycardia trial (MUSTT) registry, the all-cause 5-year mortality for patients with a QRS duration $>120 \mathrm{~ms}$ and those with $<120 \mathrm{~ms}$ was $38 \%$ and $17 \%$, respectively. ${ }^{23}$ In a pooled analysis of 10 primary prevention studies, irrespective of the QRS 
duration and aetiology of systolic dysfunction, implantation of an ICD for primary prevention provided a $7.9 \%$ absolute mortality reduction in patients with LV systolic dysfunction on optimal medical therapy ${ }^{24}$ In addition, studies such as MADIT RIT and ADVANCE III have demonstrated that if we optimise the device set-up, we can improve further on the morbidity and mortality rates of our patients. ${ }^{25} 26$

In our cohort of patients, although more patients with LVEF $\leq 35 \%$ had a QRS duration $\geq 120$ ms compared to those with LVEF $>35 \%$, the actual percentage of patients with LVEF $\leq 35 \%$ and a wide QRS duration was small at only $9 \%$. Therefore, over $90 \%$ of patients with LVEF $\leq 35 \%$ had a narrow QRS complex. Fortunately, the proposed NICE guidance has added in the caveat 'ICD if there is a high risk of SCD' in those patients with a narrow QRS complex. These high-risk features include age, sex, degree of LV dysfunction, history of MI, presence of cardiomyopathy and a range of other potential prognostic factors like B-type natriuretic peptide. NICE does not offer guidance on how these features are used when making a final clinical judgement whether the patient with a narrow QRS complex requires an ICD or not. When we used the 'history of MI' to justify use of an ICD in patients with a narrow QRS complex, the predicted ICD implantation rate was $94 \%$, similar to ESC guidance predicted implantation rates.

Currently, there are marked geographical variations in the rates of implantation of cardioverter defibrillators (ICDs), between the UK and Europe, ${ }^{8}$ and even within different regions of the UK. ${ }^{9}$ There is no evidence to suggest that disease prevalence in the UK is significantly different from that in other European countries. Compared to the rates of our European counterparts, our device implant rates are among the lowest. ${ }^{27}$ The exact causes of this inequality in the provision of device therapy remain not fully understood. One possible explanation lies in the clear differences between the ESC guidelines and NICE, particularly for complex devices (ICD), and therefore it depends on which guidance an operator chooses to implement. The north east cardiovascular network (NECVN) has agreed to follow the ESC guidance since June 2013.

When the new NICE guidance come into place, we anticipate that it would potentially increase our current ICD implantation rates and thereby close the large gap in ICD implantation rates that currently exists between the UK and our European partners, providing we agree that having a history of MI is enough to justify an ICD in patients with a narrow QRS complex, which incidentally is what the rest of the world is currently doing.

\section{CONCLUSION}

A small but significant proportion of patients with STEMIs undergoing PPCI have a severely impaired LV systolic function. A large proportion of these patients will have improved LV systolic function at 3 months. A significant number of patients continue to have severely impaired LV systolic function and a third of these patients have infarcts in territories other than anterior. There is a fivefold difference in predicted ICD implantation rates depending on which guidance is followed-NICE versus ESC. We anticipate the potential impact of the new NICE guidance on ICD implantation to be a significant increase in ICD implantation rates.

\section{LIMITATIONS}

This was a retrospective observational study. There were missing data on patients from outside our hospital catchment area (26\%). This study represents real-life data on clinical practice; the NICE guidance on 24-Holter testing and VT stimulation testing were not strictly adhered to, and therefore estimates of implantation rates could be underestimated. All hospital echocardiography reports were taken as valid and were not validated in a core laboratories.

Contributors TTP involved in the planning, conception of idea, data collection, data analysis and writing of the manuscript. SK involved in the data collection and writing of the manuscript. MMM involved in the data collection and writing of the manuscript. SM involved in the planning, data collection and review of the manuscript. VW involved in the planning and review of the manuscript. MdB involved in the planning and review of the manuscript. AT involved in the planning and review of the manuscript. SJ involved in the planning and review of the manuscript. NJL involved in the planning, review and correction of the manuscript. AJT overall content guarantor, involved in the planning and conception of the study, review and correction of the manuscript.

Competing interests TTP previously received a research grant from Medtronic Inc. NJL has received consultancy fees and honoraria from Medtronic Inc., Boston Scientific Limited and St Jude Medical Limited. He is also in receipt of a research grant from Medtronic Inc.

Provenance and peer review Not commissioned; externally peer reviewed.

Open Access This is an Open Access article distributed in accordance with the Creative Commons Attribution Non Commercial (CC BY-NC 4.0) license, which permits others to distribute, remix, adapt, build upon this work noncommercially, and license their derivative works on different terms, provided the original work is properly cited and the use is non-commercial. See: http:// creativecommons.org/licenses/by-nc/4.0/

\section{REFERENCES}

1. Gorgels AP, Gijsbers C, de Vreede-Swagemakers J, et al. Out-of-hospital cardiac arrest-the relevance of heart failure. The Maastricht Circulatory Arrest Registry. Eur Heart J 2003;24:1204-9

2. Moss AJ, Zareba W, Hall WJ, et al. Prophylactic implantation of a defibrillator in patients with myocardial infarction and reduced ejection fraction. N Engl J Med 2002;346:877-83.

3. Buxton $A E$, Lee $\mathrm{KL}$, Fisher JD, et al. A randomized study of the prevention of sudden death in patients with coronary artery disease. N Engl J Med 1999;341:1882-90.

4. Bardy GH, Lee KL, Mark DB, et al. Amiodarone or an implantable cardioverter defibrillator for congestive heart failure. $N$ Engl J Med 2005;352:225-37.

5. Zipes DP, Camm AJ, Borggrefe M, et al. ACC/AHA/ESC 2006 Guidelines for Management of Patients With Ventricular Arrhythmias and the Prevention of Sudden Cardiac DeathA Report of the American College of Cardiology/American Heart Association Task Force and the European Society of Cardiology Committee for Practice Guidelines (Writing Committee to Develop Guidelines for Management of Patients With Ventricular Arrhythmias and the Prevention of Sudden Cardiac Death). J Am Coll Cardiol 2006;48: e247-346. 
6. Keeley EC, Boura JA, Grines CL. Primary angioplasty versus intravenous thrombolytic therapy for acute myocardial infarction: a quantitative review of 23 randomised trials. Lancet 2003;361:13-20.

7. Huikuri HV, Raatikainen MP, Moerch-Joergensen R, et al. Prediction of fatal or near-fatal cardiac arrhythmia events in patients with depressed left ventricular function after an acute myocardial infarction. Eur Heart J 2009;30:689-98.

8. John Camm A, Nisam S. European utilization of the implantable defibrillator: has 10 years changed the 'enigma'? Europace 2010;12:1063-9.

9. Cunningham $A D$, Plummer $C J$, McComb JM, et al. The implantable cardioverter-defibrillator: postcode prescribing in the UK 1998-2002. Heart 2005;91:1280-3.

10. 2014. http://publications.nice.org.uk/implantable-cardioverterdefibrillators-for-arrhythmias-ta95

11. 2014. https://www.nice.org.uk/guidance/TA314/chapter/1-Guidance

12. 2013. http://www.bsecho.co.uk/home/

13. Nucifora G, Bertini M, Marsan NA, et al. Temporal evolution of left ventricular dyssynchrony after myocardial infarction: relation with changes in left ventricular systolic function. Eur Heart J Cardiovasc Imaging 2012;13:1041-6.

14. Völler $\mathrm{H}, \mathrm{Kamke} \mathrm{W}$, Klein $\mathrm{HU}$, et al. Clinical practice of defibrillator implantation after myocardial infarction: impact of implant time: results from the PreSCD II Registry. Europace 2011;13:499-508.

15. Ottervanger JP, Van't Hof AWJ, Reiffers S, et al. Long-term recovery of left ventricular function after primary angioplasty for acute myocardial infarction. Eur Heart J 2001;22:785-90.

16. Ottervanger JP, Ramdat Misier AR, Dambrink JH, et al. Mortality in patients with left ventricular ejection fraction $30 \%$ after primary percutaneous coronary intervention for ST-elevation myocardial infarction. Am J Cardiol 2007;100:793-7.

17. Huikuri HV, Raatikainen MJP, Moerch-Joergensen R, et al. Prediction of fatal or near-fatal cardiac arrhythmia events in patients with depressed left ventricular function after an acute myocardial infarction. Eur Heart J 2009;30:689-98.

18. Daubert JP, Zareba W, Hall WJ, et al. Predictive value of ventricular arrhythmia inducibility for subsequent ventricular tachycardia or ventricular fibrillation in Multicenter Automatic Defibrillator Implantation Trial (MADIT) II patients. J Am Coll Cardiol 2006;47:98-107.
19. Hohnloser SH, Kuck KH, Dorian P, et al. Prophylactic use of an implantable cardioverter defibrillator after acute myocardial infarction. N Engl J Med 2004;351:2481-8.

20. Steinbeck G, Andresen D, Seidl K, et al. Defibrillator implantation early after myocardial infarction. N Engl J Med 2009;361:1427-36.

21. Tracy CM, Epstein AE, Darbar D, et al. 2012 ACCF/AHA/HRS focused update of the 2008 guidelines for device-based therapy of cardiac rhythm abnormalitiesA report of the American College of Cardiology Foundation/American Heart Association Task Force on Practice Guidelines. J Am Coll Cardiol 2012;60:1297-313.

22. Russo AM, Stainback RF, Bailey SR, et al. ACCF/HRS/AHA/ASE/ HFSA/SCAI/SCCT/SCMR 2013 Appropriate Use Criteria for Implantable Cardioverter-Defibrillators and Cardiac Resynchronization Therapy A Report of the American College of Cardiology Foundation Appropriate Use Criteria Task Force, Heart Rhythm Society, American Heart Association, American Society of Echocardiography, Heart Failure Society of America, Society for Cardiovascular Angiography and Interventions, Society of Cardiovascular Computed Tomography, and Society for Cardiovascular Magnetic Resonance. J Am Coll Cardiol 2013;61:1318-68.

23. Buxton AE, Lee KL, DiCarlo L, et al. for the Multicenter Unsustained Tachycardia Trial Investigators. Electrophysiologic testing to identify patients with coronary artery disease who are at risk for sudden death. N Engl J Med 2000;342:1937-45.

24. Nanthakumar K, Epstein AE, Kay GN, et al. Prophylactic implantable cardioverter-defibrillator therapy in patients with left ventricular systolic dysfunctiona pooled analysis of 10 primary prevention trials. J Am Coll Cardiol 2004:44:2166-72.

25. Moss AJ, Schuger C, Beck CA, et al. Reduction in inappropriate therapy and mortality through ICD programming. N Engl J Med 2012;367:2275-83.

26. Gasparini M, Proclemer A, Klersy C, et al. Effect of long-detection interval vs standard-detection interval for implantable cardioverter-defibrillators on antitachycardia pacing and shock delivery The ADVANCE III randomized clinical trialLong-and standard-detection intervals for ICDs. JAMA 2013;309:1903-11.

27. 2013. https://nicor4.nicor.org.uk/_802571400070B77E.nsf? OpenDatabase 